\title{
Anthocyanins and Human Health: An In Vitro Investigative Approach
}

\author{
Mary Ann Lila* \\ Department of Natural Resources \& Environmental Sciences, College of Agricultural Consumer and Environmental Sciences, \\ University of Illinois, Urbana, IL 61801, USA
}

Received 2 April 2004; revised 10 May 2004; accepted 12 May 2004

\begin{abstract}
Anthocyanin pigments and associated flavonoids have demonstrated ability to protect against a myriad of human diseases, yet they have been notoriously difficult to study with regard to human health. Anthocyanins frequently interact with other phytochemicals to potentiate biological effects, thus contributions from individual components are difficult to decipher. The complex, multicomponent structure of compounds in a bioactive mixture and the degradation of flavonoids during harsh extraction procedures obscure the precise assignment of bioactivity to individual pigments. Extensive metabolic breakdown after ingestion complicates tracking of anthocyanins to assess absorption, bioavailability, and accumulation in various organs. Anthocyanin pigments and other flavonoids that are uniformly, predictably produced in rigorously controlled plant cell culture systems can be a great advantage for health and nutrition research because they are quickly, easily isolated, lack interferences found in whole fruits, can be elicited to provoke rapid and prolific accumulation, and are amenable to biolabeling so that metabolic fate can be investigated after ingestion.
\end{abstract}

\section{ANTHOCYANINS AND BIOMEDICINAL PROPERTIES}

Anthocyanins are members of the flavonoid group of phytochemicals, a group predominant in teas, honey, wines, fruits, vegetables, nuts, olive oil, cocoa, and cereals. The flavonoids, perhaps the most important single group of phenolics in foods, comprise a group of over 4000 $\mathrm{C}_{15}$ aromatic plant compounds with multiple substitution patterns (www.nal.usda.gov/fnic/foodcomp/index.html). The primary players in this group include the anthocyanins (eg, cyanidin, pelargonidin, petunidin), the flavonols (quercetin, kaempferol), flavones (luteolin, apigenin), flavanones (myricetin, naringin, hesperetin, naringenin), flavan-3-ols (catechin, epicatechin, gallocatechin), and, although sometimes classified separately, the isoflavones (genistein, daidzein). Phytochemicals in this class are frequently referred to as bioflavonoids due to their multifaceted roles in human health maintenance, and anthocyanins in food are typically ingested as components of complex mixtures of flavonoid components. Daily intake is estimated from $500 \mathrm{mg}$ to $1 \mathrm{~g}$, but can be several $\mathrm{g} / \mathrm{d}$ if an individual is consuming flavonoid supplements (grape seed extract, ginkgo biloba, or pycnogenol; see, eg, [1]).

The colorful anthocyanins are the most recognized, visible members of the bioflavonoid phytochemicals. The free-radical scavenging and antioxidant capacities of anthocyanin pigments are the most highly publicized of the modus operandi used by these pigments to intervene with human therapeutic targets, but, in fact, research clearly suggests that other mechanisms of action are also responsible for observed health benefits $[2,3,4,5]$. Anthocyanin isolates and anthocyanin-rich mixtures of bioflavonoids may provide protection from DNA cleavage, estrogenic activity (altering development of hormone-dependent disease symptoms), enzyme inhibition, boosting production of cytokines (thus regulating immune responses), anti-inflammatory activity, lipid peroxidation, decreasing capillary permeability and fragility, and membrane strengthening $[6,7,8,9,10]$. The chemical structure (position, number, and types of substitutions) of the individual anthocyanin molecule also has a bearing on the degree to which anthocyanins exert their bioactive properties $[11,12]$ and the structure/function relationships also influence the intracellular localization of the pigments [7]. The anthocyanin literature includes some controversy over the relative contributions of glycosylated anthocyanins versus aglycones in terms of bioavailability and bioactive potential $[7,13,14,15,16]$. Originally, it was assumed that only aglycones could enter the circulation circuit, however, absorption and metabolism of anthocyanin glycosides has now been demonstrated. The nature of the sugar conjugate and the aglycone are important determinants of anthocyanin absorption and excretion in both humans and rats [15].

The roles of anthocyanin pigments as medicinal agents have been well-accepted dogma in folk medicine throughout the world, and, in fact, these pigments are linked to an amazingly broad-based range of health benefits. For example, anthocyanins from Hibiscus sp have 
historically been used in remedies for liver disfunction and hypertension; and bilberry (Vaccinium) anthocyanins have an anecdotal history of use for vision disorders, microbial infections, diarrhea, and diverse other health disorders $[17,18,19]$. But while the use of anthocyanins for therapeutic purposes has long been supported by both anecdotal and epidemiological evidence, it is only in recent years that some of the specific, measurable pharmacological properties of isolated anthocyanin pigments have been conclusively verified by rigorously controlled in vitro, in vivo, or clinical research trials [4]. In many other cases, the exact roles of the anthocyanins in human health maintenance versus other phytochemicals in a complex mixture from a fruit extract or whole food have not been completely sorted out. In fact, some reports suggest that anthocyanin activity is potentiated when delivered in mixtures [20,21, 22].

For example, visual acuity can be markedly improved through administration of anthocyanin pigments to animal and human subjects, and the role of these pigments in enhancing night vision or overall vision has been particularly well documented [23]. Oral intake of anthocyanosides from black currants resulted in significantly improved night vision adaptation in human subjects [24], and similar benefits were gained after administration of anthocyanins from bilberries [25]. Three anthocyanins from black currant stimulated regeneration of rhodopsin (a G-protein-coupled receptor localized in the retina of the eye), and formation of a regeneration intermediate was accelerated by cyanidin 3-rutinoside [26]. These studies strongly suggest that enhancement of rhodopsin regeneration is at least one mechanism by which anthocyanins enhance visual acuity.

In both in vitro and in vivo research trials, anthocyanins have demonstrated marked ability to reduce cancer cell proliferation and to inhibit tumor formation $[27,28,29,30]$. The capacity of anthocyanin pigments to interfere with the process of carcinogenesis seems to be linked to multiple potential mechanisms of action including inhibition of cyclooxygenase enzymes and potent antioxidant potential. Hou et al [20] revealed that anthocyanins inhibit tumorigenesis by blocking activation of a mitogen-activated protein kinase pathway. This report provided the first indication of a molecular basis for why anthocyanins demonstrate anticarcinogenic properties. In other research, fruit extracts with significant anthocyanin concentrations proved to be effective against various stages of carcinogenesis [18, 28, 31, 32], but the individual role of anthocyanins versus other components was not determined, in part because the anthocyanins were too easily degraded during bioassays if separated from stabilizing cofactors such as other phenolic constituents [33].

The role of anthocyanins in cardiovascular disease protection is strongly linked to oxidative stress protection. Since endothelial dysfunction is involved in initiation and development of vascular disease, four anthocyanins isolated from elderberries were incorporated into the plasma lemma and cytosol of endothelial cells to directly examine the protective roles [34]. These tests demonstrated not only that anthocyanins could be directly incorporated into endothelial cells, but that significant oxidative stress protection was the result. Delphinidin, but not malvidin or cyanidin, provided endothelium-dependent vasorelaxation in the rat aorta, providing a pharmacological benefit comparable to red wine polyphenolics [35]. In a rat model, little influence of feeding purified anthocyanins (cyanidin 3-O-glucoside) or anthocyanin-rich extracts from elderberry or blackcurrant could be detected on cholesterol levels or fatty acid patterns in liver, but the pigments were capable of sparing vitamin E [36]. Crude anthocyanin extracts from bilberry have been administered both orally and via injection to reduce capillary permeability [13]. In other research related to cardiovascular impairment, the roles of anthocyanin pigments versus other flavonoids delivered in the phytochemical extract have not been completely sorted out. Protection from heart attacks through administration of grape juice or wine was strongly tied to the ability of the anthocyaninrich products to reduce inflammation and enhance capillary strength and permeability, and to inhibit platelet formation and enhance nitric oxide (NO) release [37]. Similarly, delivery of a black currant concentrate with intense anthocyanin content caused endothelium-dependent vasorelaxation in rat aorta rings in vitro [38]. The mechanism of vasorelaxation was attributed to increased levels of NO production, but the active compounds in the concentrate were not isolated. When rats were pretreated to create increased susceptibility to oxidative damage, then fed anthocyanin-rich extracts, significant reduction in indices of lipid peroxidation and DNA damage resulted [9]. Ingestion of these extracts, which contained mixtures of delphinidin, cyanidin, petunidin, peonidin, and malvidin in the 3-glucopyranoside forms, also increased plasma antioxidant capacity.

Tsuda et al [4] recently provided evidence that anthocyanins extracted from purple corn, when provided to mice in tandem with a high-fat diet, effectively inhibited both body weight and adipose tissue increases. Typical symptoms of hyperglycemia, hyperinsulinemia, and hyperleptinemia provoked by a high-fat diet did not occur when mice also ingested isolated anthocyanins. The experiments suggest that anthocyanins, as a functional food component, can aid in the prevention of obesity and diabetes.

Anthocyanins have been credited with capacity to modulate cognitive and motor function, to enhance memory, and to have a role in preventing age-related declines in neural function. Cho et al [39] reported that administration of isolated semipurified anthocyanins from purple sweet potato enhanced cognitive performance as assessed by passive avoidance tests in ethanol-treated mice, and also effectively inhibited lipid peroxidation in rat brain tissues. By administering blueberry extracts with significant anthocyanin content (but not purified pigments), it was noted that the blueberry-supplemented 
diets led to effective reversal of age-related deficits in various neural and behavioral parameters (memory and motor functions) [40]. Further investigations by this laboratory team demonstrated that anthocyanins (in particular, cyanidin-3-sambubioside-5-glucoside and cyanidin3, 5-diglucoside) were highly bioavailable in endothelial cells, which was linked to their roles in prevention of atherosclerosis and neurodegenerative disorders [34, 41].

Anthocyanins exerted multiple protective effects against pleurisy in a rat model and were capable of attenuating inflammation. Anthocyanin treatment also downregulated expression of enzymes involved in inflammation in the lung [10]. The antimicrobial activity of anthocyanins in general has been well established, including significant inhibition of aflatoxin biosynthesis [42]. The experimental evidence demonstrating anthocyanin benefits for diabetes and pancreatic disorders has also accumulated in recent years, and again the efficacy is attributed to the multiple, simultaneous biological effects these pigments cause in the body, including prevention of generation of free radicals, decreased lipid peroxidation, reduced pancreatic swelling, and decreased blood sugar concentrations in urine and blood serum $[43,44]$.

\section{THE ANTHOCYANIN ENIGMA}

An enigma is defined as anything that perplexes because it is inexplicable, hidden, or obscure; something that serves as a puzzle to be solved. The whole realm of anthocyanin consumption and human health fits into this definition, because several aspects of anthocyanin's pharmacological roles have remained elusive to the scientist. In most of the interventions of anthocyanins in human health, details on the mechanisms of action for bioactivity, uptake, absorption, bioavailability, whole body distribution, and tissue localization are still not fully elucidated.

There are at least four primary obstacles that have impeded the formulation, by medical professionals, of robust dietary or prescriptive guidelines on consumption of anthocyanins.

Probably the most complicated piece of the puzzle is that, in terms of biological activity in the human body, an anthocyanin pigment is (almost) never acting independently. Typically, anthocyanins and other flavonoid components, or anthocyanins and other nonflavonoid phytochemicals, are interacting together in order to provide full potency. Interactions between phytochemicals within a plant are a key evolutionary strategy for the host plant. There are over 4000 flavonoids described, with multiple substitution patterns and often large complex structures in the mixtures. Bioflavonoids like anthocyanins occur in mixtures within edible foods and are ingested in mixtures. Any plant containing anthocyanins includes a complex phytochemical cocktail. The anthocyanins and related flavonoids are secondary products typically produced by plants as defensive, protective, or attractive agents, and it makes good evolutionary sense for the plant to use a variety of strategies and multiple fronts of attack to accomplish these functions, rather than single compounds to which a pathogen or predator could become resistant. This same multiplicity in bioactive phytochemical synthesis is also a bonus for animals and humans who ingest the plant material donors, and benefit from the interaction of the flavonoids with therapeutic targets. When the interactions between co-occurring phytochemicals are positive (eg, additive effects or synergies), they are called potentiating interactions. In other cases, components in the donor plant can actually inhibit the bioactivity of the flavonoid compound (eg, pectin interference with antioxidant capacity in in vitro assays), and in other cases, concomitant compounds which are not themselves bioactive may work together with a bioflavonoid to enhance bioavailability or absorption. Synergy among flavonoids including anthocyanins has been cited as responsible for antiplatelet activity of red wine and grape juice, with strong interactions between components of grape skin and grape seed required to potentiate antiplatelet activity in human and animal systems [45]. Co-occurring flavonoids working synergistically to antagonize hydrogen peroxide formation are most effective in depressing platelet function [46]. Traditional bioprospecting approaches, which search for single purified plant-derived compounds as a means of drug discovery, will not capture the full potency of a plant extract when multiple potentiating interactions are responsible for bioactivity.

Another common well-recognized handicap to scientists exploring the bioactive properties of the flavonoids, and the second part of the anthocyanin puzzle, is the fact that these phytochemicals can be of an evanescent nature [33]. The susceptibility of anthocyanins to oxidation and degradation is one of the concerns of food processors who wish to maximize the shelf life of products enhanced with natural pigment colors. In particular, many of the classic phytochemical methods (including column chromatography), used to extract from plant tissues and fractionate components out of a crude extract, can degrade anthocyanins and/or inactivate them during purification steps. As a result, research that aims to identify bioactive entities and gauge potencies of extracts can easily fail to assess the actual sources of biological activity in situ.

Strict attention to the ephemeral nature of some flavonoid constituents in berries (especially during extraction/fractionation sequences) led to the adaptation of a vacuum chromatography technique in our laboratory, which was designed to (as much as feasible) preserve the integrity of the compounds and keep natural mixtures intact until final separation for purposes of identification $[32,47]$. Using whole individually-quick-frozen berries as a starting point, fruits are extracted in a Waring blender with $70 \%$ aqueous acetone ( $\sim 2 \mathrm{~L}$ solvent $\mathrm{kg}^{-1}$ fruit) then filtered through cheesecloth. Acetone is removed from the filtrate under vacuum in a $40^{\circ} \mathrm{C}$ water bath, and water is then removed by lyophilization, resulting in a dark purple powder. A portion of the crude dry extract is then 
redissolved in water and poured over a Toyopearl resin polymer column for vacuum chromatography. Vacuum chromatography on Toyopearl with a series of solvents (water, $50 \% \mathrm{MeOH}, 100 \% \mathrm{MeOH}, 100 \%$ acetone, and $50 \%$ acetone) elutes $5 \mathrm{TP}$ fractions which are then concentrated under vacuum, and water is removed by lyophilization. Sugars are very quickly and efficiently removed in the first fraction, which greatly simplifies the handling and analysis of remaining fractions.

Once bioactive fractions are identified, a second, third, and subsequent rounds of separation are accomplished on silica gel, also by vacuum, sometimes open column gravity chromatography. At each step of the procedure, isolated mixtures are compared using silica gel thin layer chromatography and 2 spray reagents (vanillin- $\mathrm{HCl}$ and dichromate reagent) in order to gauge the composition and number of components in each fraction. In general, this fractionation strategy has permitted rapid separation of relatively large volumes of extract, with less exposure to damaging and expensive solvents, less exposure to column support materials and air, minimal losses, and reliable separation of flavonoids. In tandem with all of the fractionations is a consistent sequence of bioassays (for multiple stages of carcinogenesis) because the fractionation scheme is bioactivity-guided. As fractions become more highly purified, analysis with HPLC, HPLC-MS, and NMR can be used to conclusively determine the origins of the bioactivity.

A third piece of the puzzle is the inducible nature of many of the bioactive flavonoids including anthocyanins. As is true of a plethora of secondary plant products, the initial production and accumulation of phytochemicals is triggered by physical or chemical microenvironmental triggers, usually a stress factor. The genes responsible for flavonoid synthesis are highly inducible. As such, a researcher intent on maximizing production of anthocyanin pigments must recognize the induction factors and deliberately elicit production of bioactive flavonoids by providing these stimuli to the plant material of interest. Elicitation mimics stresses that provoke secondary product formation in nature, and activates otherwise dormant biochemical pathways. This triggering of productivity can, of course, be very difficult to accomplish in a field setting, but can be accomplished reliably in controlled growth facilities.

The final puzzle piece in the "anthocyanin enigma" is the inability of the scientist or medicinal practitioner to track metabolic progress of anthocyanins after ingestion, due to the plethora of metabolic breakdown products that are rapidly produced in situ. There is substantial current interest in the quest to follow the transport of bioflavonoids through the body, to determine absorption and bioavailability, and to see where breakdown products accumulate and for how long. However, since these phytochemicals are highly metabolized after consumption of anthocyanin-rich foods or supplements, metabolic tracking has not been possible. Despite active research and increasing interest in the realm of natural products and health maintenance, there is a paucity of information on the absorption, biodistribution, and metabolism of anthocyanins and interacting flavonoids. Various plant secondary products have been implicated in the promotion of good health or the prevention of disease in humans, but little is known about the way they are absorbed in the gut, or in which tissues they are deposited throughout the body. While these issues could be studied if the phytochemicals were isotopically labeled, generating labeled molecules often is problematic because many compounds of interest can be synthesized only in planta at present.

\section{IN VITRO ANTHOCYANIN PRODUCTION SYSTEMS}

The development and optimization of plant cell culture systems which reliably and predictably synthesize anthocyanins in a controlled environment has provided a unique and useful model for in-depth research on anthocyanins, and has helped at least in part to circumvent the obstacles presented in all four cases of the "anthocyanin enigma" as described above. Callus and cell suspension cultures from a wide and diverse range of plant genera have been cultivated to produce anthocyanin pigments in vitro $[48,49,50,51,52,53,54,55,56]$. In most of these past reports, the overall goal of the plant cell culture production system was to explore an alternative resource for natural plant pigments, for possible use as food colorants. More recently, some anthocyaninproducing plant species have been intensively cultured in vitro in order to harvest the bioactive pigments and related phytochemicals as medicinally-active compounds $[47,57,58,59]$. By controlling both the physical and the chemical microenvironment of the plant cell cultures, anthocyanin production could in many cases be boosted to higher concentrations than available in the parent plant in vivo. Some of the most intensively-researched cell culture production systems used selections from the genus Vitis (grape), where scaled-up bioreactor-based systems approached semicommercial productivity $[60,61]$. The cell culture systems can be quite stable, and many have been selected for high anthocyanin yield and lack of dependence on irradiance. Anthocyanin profiles from cell cultures do not necessarily mirror the profiles from the parent plant, and isolation of pigments from the simplified cell culture tissues is substantially more streamlined than from complex fruit or vegetative tissues $[47,53,58]$. This simplicity can be a particular advantage for investigation of the health properties of bioflavonoids including anthocyanins.

In most cases, the systems begin with vegetative plant materials (leaves, petioles, stems) and not fruit tissues. Explants from in vivo plants are surface-disinfested and introduced into cell culture to produce rapidly proliferating callus, then cell suspension cultures, which are eventually induced to produce flavonoids (usually with a trigger such as light, elevated carbohydrate, changed nitrogen profile, 
or elicitation with a fungal extract or other chemical elicitor).

Because cell culture anthocyanin production systems are comprised of simple tissues which can be engineered to reliably and predictably accumulate pigment, these systems circumvent many of the obstacles in the anthocyanin enigma. Interactions between potentiating phytochemicals are still in force in cell culture systems, but because the tissues are much simpler to extract, the nature of phytochemical interactions is much easier to sort out and to quantitatively test. Cell cultures permit rapid and efficient isolation without many of the interfering compounds (pectins, excess polysaccharides, enzymes) that can complicate extraction or bioassay from fruits [18]. Aqueous extracts of an anthocyanin-producing sweet potato line exhibited higher potency (antiproliferative and antimutagenic potential) than extract from field-grown crops [58]. Similarly, when antioxidant capacity of cell cultures and various fruit extracts were compared side by side in a galvinoxyl free radical assay, the potency of the cell culture extract was substantially higher than that of all fruit extracts, and only grape seed proanthocyanidins exhibited higher activity [47]. Because these other substances are not present, the flavonoids are much easier to isolate without the degradation that can occur rapidly when isolating slowly from complex, recalcitrant fruit or vegetative tissues.

While the flavonoid content of a fruit may comprise only $1 \%$ or less of the total substance, a cell culture can be crafted to accumulate much higher concentrations of flavonoids, in the range of $20 \%-30 \%$ by volume. Many flavonoids, in particular, high-molecular weight proanthocyanidin oligomers or complex anthocyanin isomers, are either not available commercially or prohibitively expensive. By producing these phytochemicals in volume in cell cultures, a source of ready standards is available for testing unknowns [32].

Cell cultures are a superb model system for testing the effects of elicitation on the inducible bioflavonoid genes, which is a means of resolving yet another aspect of the anthocyanin enigma. In fact, elicitation of cell cultures (using chemical or environmental triggers to production) is a recognized and efficient means of maximizing anthocyanin pigment towards commercialization of product recovery $[55,56,62,63]$, and since the in vitro production environment is rigorously controlled, investigators have the opportunity to test multiple elicitation triggers without interference from uncontrolled environmental conditions in field settings.

Perhaps the most significant advantage to investigation using in vitro anthocyanin-accumulating cell cultures is that the cultures can serve as a vehicle for delivery of isotopic labels (13C or 14C) to the metabolizing cells while the pigments are being biosynthesized $[59,64,65]$. By using a radioisotope-labeled source of compounds, an administered phytochemical can be included in a defined diet and can be discerned from preexisting, endogenous sources of the same phytochemical or breakdown product. These large molecules must be synthesized in planta. In this emerging research area, radiolabel or isotopic label has been introduced to metabolizing cell cultures using a carbohydrate source (sucrose or glucose) or a precursor which is much further along the metabolic pathway to anthocyanin synthesis, such as phenylalanine. Levels of incorporation range between $15 \%$ and $30 \%$, and levels achieved now allow organ and neuronal localization of the ${ }^{14} \mathrm{C}$-labeled compounds and monitoring using autoradiography and scintillation counting. Accelerator mass spectrometry (AMS) technology will even permit monitoring of small levels in human systems. With these innovations, it is clear that the effective use of cell-cultureproduced anthocyanins can now elucidate previously hidden roles of anthocyanin pigments in human health and metabolism.

\section{REFERENCES}

[1] Skibola C, Smith M. Potential health impacts of excessive flavonoid intake. Free Radic Biol Med. 2000;29(3-4):375-383.

[2] Tsuda T, Shiga K, Ohshima K, Kawakishi S, Osawa T. Inhibition of lipid peroxidation and the active oxygen radical scavenging effect of anthocyanin pigments isolated from Phaseolus vulgaris L. Biochem Pharmacol. 1996;52(7):1033-1039.

[3] Tsuda T, Horio F, Osawa T. Cyanidin 3-O-beta-Dglucoside suppresses nitric oxide production during a zymosan treatment in rats. J Nutr Sci Vitaminol (Tokyo). 2002;48(4):305-310.

[4] Tsuda T, Horio F, Uchida K, Aoki H, Osawa T. Dietary cyanidin 3-O-beta-D-glucoside-rich purple corn color prevents obesity and ameliorates hyperglycemia in mice. J Nutr. 2003;133(7):2125-2130.

[5] Wang S, Jiao H. Scavenging capacity of berry crops on superoxide radicals, hydrogen peroxide, hydroxyl radicals, and singlet oxygen. J Agric Food Chem. 2000;48(11):5677-5684.

[6] Acquaviva R, Russo A, Galvano F, et al. Cyanidin and cyanidin 3-O-beta-D-glucoside as DNA cleavage protectors and antioxidants. Cell Biol Toxicol. 2003;19(4):243-252.

[7] Lazze M, Pizzala R, Savio M, Stivala L, Prosperi E, Bianchi L. Anthocyanins protect against DNA damage induced by tert-butyl-hydroperoxide in rat smooth muscle and hepatoma cells. Mutat Res. 2003;535(1):103-115.

[8] Lefevre M, Howard L, Most M, Ju Z, Delany J. Microarray analysis of the effects of grape anthocyanins on hepatic gene expression in mice. FASEB J. 2004;18:A851.

[9] Ramirez-Tortosa C, Andersen O, Gardner P, et al. Anthocyanin-rich extract decreases indices of lipid peroxidation and DNA damage in vitamin Edepleted rats. Free Radic Biol Med. 2001;31(9):10331037. 
[10] Rossi A, Serraino I, Dugo P, et al. Protective effects of anthocyanins from blackberry in a rat model of acute lung inflammation. Free Radic Res. 2003;37(8):891-900.

[11] Prior R, Cao G, Martin A, et al. Antioxidant capacity as influenced by total phenolic and anthocyanin content, maturity, and variety of Vaccinium species. J Agric Food Chem. 1998;46(7):2686-2693.

[12] Russo A, Acquaviva R, Campisi A, et al. Bioflavonoids as antiradicals, antioxidants and DNA cleavage protectors. Cell Biol Toxicol. 2000;16(2):9198.

[13] Kong JM, Chia LS, Goh NK, Chia TF, Brouillard R. Analysis and biological activities of anthocyanins. Phytochemistry. 2003;64(5):923-933.

[14] Matsumoto H, Nakamura Y, Hirayama M, Yoshiki Y, Okubo K. Antioxidant activity of black currant anthocyanin aglycons and their glycosides measured by chemiluminescence in a neutral $\mathrm{pH}$ region and in human plasma. J Agric Food Chem. 2002;50(18):5034-5037.

[15] McGhie T, Ainge G, Barnett L, Cooney J, Jensen D. Anthocyanin glycosides from berry fruit are absorbed and excreted unmetabolized by both humans and rats. J Agric Food Chem. 2003;51(16): 4539-4548.

[16] Miyazawa T, Nakagawa K, Kudo M, Muraishi K, Someya K. Direct intestinal absorption of red fruit anthocyanins, cyanidin-3-glucoside and cyanidin3,5-diglucoside, into rats and humans. J Agric Food Chem. 1999;47(3):1083-1091.

[17] Rice-Evans C, Packer L, eds. Flavonoids in Health and Disease. NY: Marcel Dekker; 1998.

[18] Smith M, Marley K, Seigler D, Singletary K, Meline B. Bioactive properties of wild blueberry fruits. $J$ Food Sci. 2000;65:352-356.

[19] Wang C, Wang J, Lin W, Chu C, Chou F, Tseng T. Protective effect of Hibiscus anthocyanins against tert-butyl hydroperoxide-induced hepatic toxicity in rats. Food Chem Toxicol. 2000;38(5):411-416.

[20] Hou DX, Kai K, Li JJ, et al. Anthocyanidins inhibit activator protein 1 activity and cell transformation: structure-activity relationship and molecular mechanisms. Carcinogenesis. 2004;25(1):29-36.

[21] Liu R. Health benefits of fruit and vegetables are from additive and synergistic combinations of phytochemicals. Am J Clin Nutr. 2003;78(suppl 3):517S520S.

[22] Stintzing F, Stintzing A, Carle R, Frei B, Wrolstad R. Color and antioxidant properties of cyanidinbased anthocyanin pigments. J Agric Food Chem. 2002;50(21):6172-6181.

[23] Matsumoto H, Inaba H, Kishi M, Tominaga S, Hirayama $M$, Tsuda $T$. Orally administered delphinidin 3-rutinoside and cyanidin 3-rutinoside are directly absorbed in rats and humans and appear in the blood as the intact forms. J Agric Food Chem. 2001;49(3):1546-1551.
[24] Nakaishi H, Matsumoto H, Tominaga S, Hirayama M. Effects of black currant anthocyanoside intake on dark adaptation and VDT work-induced transient refractive alteration in healthy humans. Altern Med Rev. 2000;5(6):553-562.

[25] Muth ER, Laurent J, Jasper P. The effect of bilberry nutritional supplementation on night visual acuity and contrast sensitivity. Altern Med Rev. 2000;5(2):164-173.

[26] Matsumoto H, Nakamura Y, Tachibanaki S, Kawamura S, Hirayama M. Stimulatory effect of cyanidin 3-glycosides on the regeneration of rhodopsin. J Agric Food Chem. 2003;51(12):3560-3563.

[27] Hou DX. Potential mechanisms of cancer chemoprevention by anthocyanins. Curr Mol Med. 2003;3(2): 149-159.

[28] Kang S, Seeram N, Nair M, Bourquin L. Tart cherry anthocyanins inhibit tumor development in $\mathrm{Apc}(\mathrm{Min})$ mice and reduce proliferation of human colon cancer cells. Cancer Lett. 2003; 194(1):13-19.

[29] Koide T, Hashimoto Y, Kamei H, Kojima T, Hasegawa M, Terabe K. Antitumor effect of anthocyanin fractions extracted from red soybeans and red beans in vitro and in vivo. Cancer Biother Radiopharm. 1997;12(4):277-280.

[30] Meiers S, Kemeny M, Weyand U, Gastpar R, von Angerer E, Marko D. The anthocyanidins cyanidin and delphinidin are potent inhibitors of the epidermal growth-factor receptor. J Agric Food Chem. 2001;49(2):958-962.

[31] Bomser J, Madhavi D, Singletary K, Smith MA. In vitro anticancer activity of fruit extracts from Vaccinium species. Planta Med. 1996;62(3):212-216.

[32] Kandil F, Song L, Pezzuto J, Seigler D, Smith MA. Isolation of oligomeric proanthocyanidins from flavonoid-producing cell cultures. In Vitro Cell Dev Biol Plant. 2000;36:492-500.

[33] Mazza G, Miniati E. Anthocyanins in Fruits, Vegetables, and Grains. Boca Raton, Fla: CRC Press; 1993.

[34] Youdim K, Martin A, Joseph J. Incorporation of the elderberry anthocyanins by endothelial cells increases protection against oxidative stress. Free Radic Biol Med. 2000;29(1):51-60.

[35] Andriambeloson E, Magnier C, Haan-Archipoff G, et al. Natural dietary polyphenolic compounds cause endothelium-dependent vasorelaxation in rat thoracic aorta. J Nutr. 1998;128(12):2324-2333.

[36] Frank J, Kamal-Eldin A, Lundh T, Maatta K, Torronen R, Vessby B. Effects of dietary anthocyanins on tocopherols and lipids in rats. J Agric Food Chem. 2002;50(25):7226-7230.

[37] Folts J. Antithrombotic potential of grape juice and red wine for preventing heart attacks. Pharm Biol. 1998;36(suppl):21-27.

[38] Nakamura Y, Matsumoto H, Todoki K. Endothelium-dependent vasorelaxation induced by black 
currant concentrate in rat thoracic aorta. Jpn J Pharmacol. 2002;89(1):29-35.

[39] Cho J, Kang J, Long P, Jing J, Back Y, Chung K. Antioxidant and memory enhancing effects of purple sweet potato anthocyanin and Cordyceps mushroom extract. Arch Pharm Res. 2003;26(10):821-825.

[40] Joseph J, Shukitt-Hale B, Denisova N, et al. Reversals of age-related declines in neuronal signal transduction, cognitive, and motor behavioral deficits with blueberry, spinach, or strawberry dietary supplementation. J Neurosci. 1999;19(18):8114-8121.

[41] Youdim K, McDonald J, Kalt W, Joseph J. Potential role of dietary flavonoids in reducing microvascular endothelium vulnerability to oxidative and inflammatory insults. J Nutr Biochem. 2002;13(5):282-288.

[42] Norton R. Inhibition of aflatoxin B(1) biosynthesis in Aspergillus flavus by anthocyanidins and related flavonoids. J Agric Food Chem. 1999;47(3):12301235.

[43] Jankowski A, Jankowska B, Niedworok J. The influence of Aronia melanocapra in experimental pancreatitis. Pol Merkuriusz Lek. 2000;8(48):395-398.

[44] Jankowski A, Jankowska B, Niedworok J. The effect of anthocyanin dye from grapes on experimental diabetes. Folia Med Cracov. 2000;41(3-4):5-15.

[45] Shanmuganayagam D, Beahm M, Osman H, Krueger C, Reed J, Folts J. Grape seed and grape skin extracts elicit a greater antiplatelet effect when used in combination than when used individually in dogs and humans. J Nutr. 2002;132(12):3592-3598.

[46] Pignatelli P, Pulcinelli F, Celestini A, et al. The flavonoids quercetin and catechin synergistically inhibit platelet function by antagonizing the intracellular production of hydrogen peroxide. Am J Clin Nutr. 2000;72(5):1150-1155.

[47] Kandil F, Smith M, Rogers R, et al. Composition of a chemopreventive proanthocyanidin-rich fraction from cranberry fruits responsible for the inhibition of 12-O-tetradecanoyl phorbol-13-acetate (TPA)-induced ornithine decarboxylase (ODC) activity. J Agric Food Chem. 2002;50(5):1063-1069.

[48] Callebaut A, Declere M, Vandermeren K. Ajuga reptans (Bugle). In vitro production of anthocyanins. In: Bajaj YPS, ed. Biotechnology in Agriculture and Forestry 24. Medicinal and Aromatic Plants V. Berlin: Springer-Verlag; 1993:1-22.

[49] Cormier F, Crevier H, Do C. Effects of sucrose concentration on the accumulation of anthocyanins ingrape (Vitis vinifera L.) cell suspension. Can J Bot. 1990;68:1822-1825.

[50] Ilan A, Dougall D. The effect of growth retardants on anthocyanin production in carrot cell suspension cultures. Plant Cell Rep. 1992;11(5-6):304-309.

[51] Kobayashi Y, Akita M, Sakamoto K, et al. Largescale production of anthocyanin by Aralia cordata cell suspension cultures. Appl Microbiol Biotechnol. 1993;40(2-3):215-218.

[52] Konczak-Islam I, Yoshinaga M, Nakatani M, Ter- ahara N, Yamakawa O. Establishment and characteristics of an anthocyanin-producing cell line from sweet potato storage root. Plant Cell Rep. 2000;19:472-477.

[53] Meyer J, Pépin MF, Smith M. Anthocyanin production from Vaccinium pahalae: limitations of the physical microenvironment. J Biotechnol. 2002; 93(1):45-57.

[54] Mizukami H. Hibiscus sabdariffa L. (Roselle): In vitro culture and the production of anthocyanins. In: Bajaj YPS, ed. Biotechnology in Agriculture and Forestry 24. Medicinal and Aromatic Plants V. Berlin: Springer-Verlag; 1993:218-228.

[55] Zhong J, Yoshida M, Fujiyama D, Seki T, Yoshida T. Enhancement of anthocyanin production by Perilla frutescens cells in a stirred bioreactor with internal light irradiation. J Ferment Bioeng. 1993;75(4):299303.

[56] Zubko M, Schmeer K, Gläßgen W, Bayer E, Seitz H. Selection of anthocyanin-accumulating potato (Solanum tuberosum L.) cell lines from calli derived from seedlings germinated from gamma-irradiated seeds. Plant Cell Rep. 1993;12:555-558.

[57] Pezet R, Perret C, Jean-Denis J, Tabacchi R, Gindro K, Viret O. Delta-viniferin, a resveratrol dehydrodimer: one of the major stilbenes synthesized by stressed grapevine leaves. J Agric Food Chem. 2003;51(18):5488-5492.

[58] Konczak-Islam I, Yoshimoto M, Hou D, Terahara N, Yamakawa O. Potential chemopreventive properties of anthocyanin-rich aqueous extracts from in vitro produced tissue of sweetpotato (Ipomoea batatas L.). J Agric Food Chem. 2003;51(20):5916-5922.

[59] Yousef G, Seigler D, Grusak M, et al. Biosynthesis and characterization of ${ }^{14} \mathrm{C}$-enriched flavonoid fractions from plant cell suspension cultures. J Agric Food Chem. 2004;52(5):1138-1145.

[60] Payne G, Bringi V, Prince C, Shuler M. Plant Cell and Tissue Culture in Liquid Systems. NY: Hanser; 1992.

[61] Shuler M, Hirasuna T, Prince C, Bringi V. Bioreactor considerations for producing flavors and pigments from plant tissue culture. In: Schwarzberg H, Rao M, eds. Bioprocess and Food Process Engineering. New York: Marcel-Dekker; 1990:45-66.

[62] Hirner A, Veit S, Seitz H. Regulation of anthocyanin biosynthesis in UV-A-irradiated cell cultures of carrot and in organs of intact carrot plants. Plant Sci. 2001;161(2):315-322.

[63] Mori T, Sakurai M, Sakuta M. Effects of conditioned medium on activities of PAL, CHS, DAHP synthase (DS-Co and DS-Mn) and anthocyanin production in suspension cultures of Fragaria ananassa. Plant Sci. 2001;160(2):335-360.

[64] Grusak M, Rogers R, Yousef G, Erdman J Jr, Lila M. An enclosed-chamber labeling system for the safe ${ }^{14} \mathrm{C}$-enrichment of phytochemicals in plant cell suspension cultures. In Vitro Cell Dev Biol Plant. 2004;40:80-85. 
[65] Vitrac X, Desmouliere A, Brouillaud B, et al. Distribution of $\left[{ }^{14} \mathrm{C}\right]$-trans-resveratrol, a cancer chemopreventive polyphenol, in mouse tissues after oral administration. Life Sci. 2003;72(20):2219-2233.

* E-mail: imagemal@uiuc.edu

Fax:+1 217244 3469; Tel:+1 2173335154 

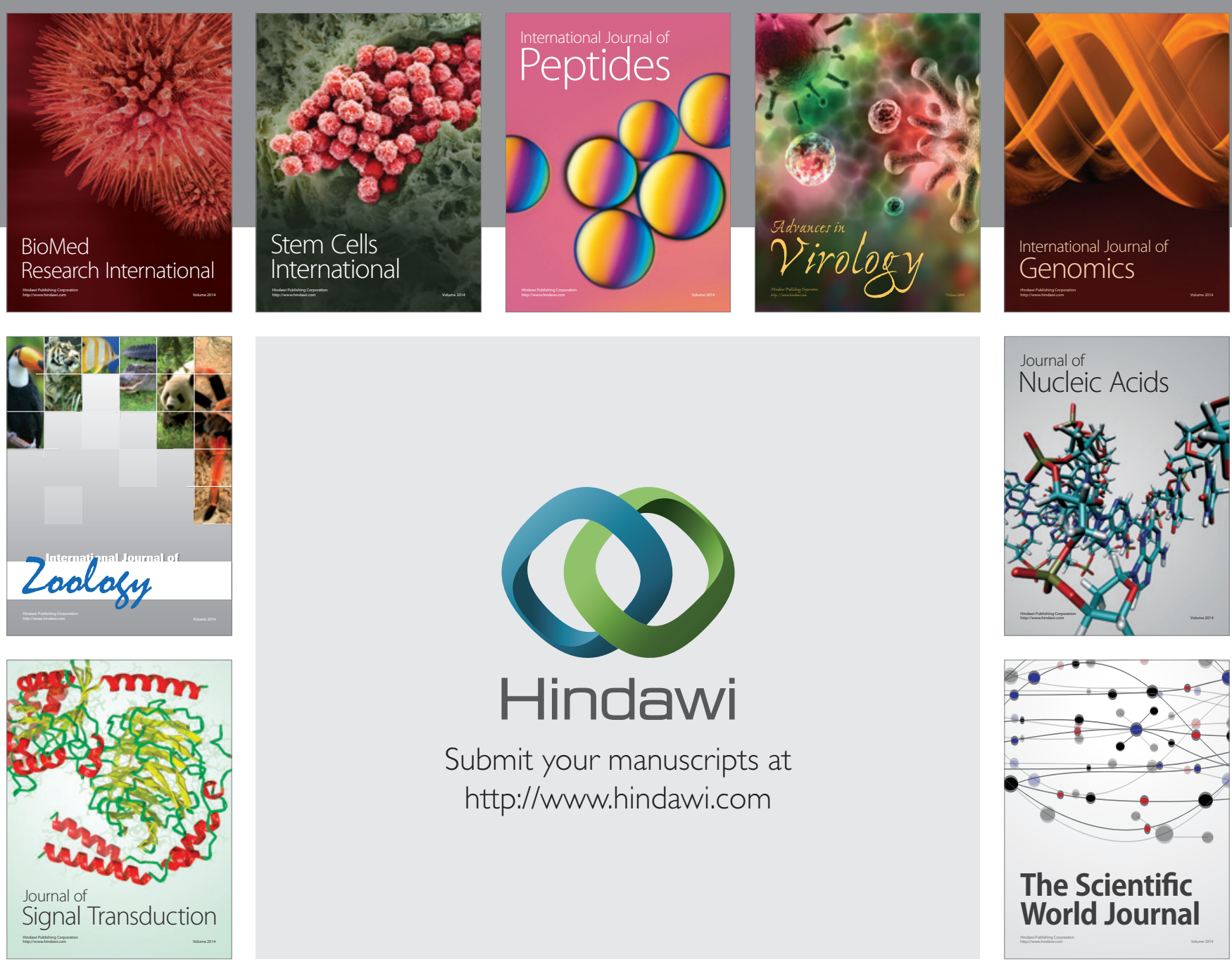

Submit your manuscripts at

http://www.hindawi.com
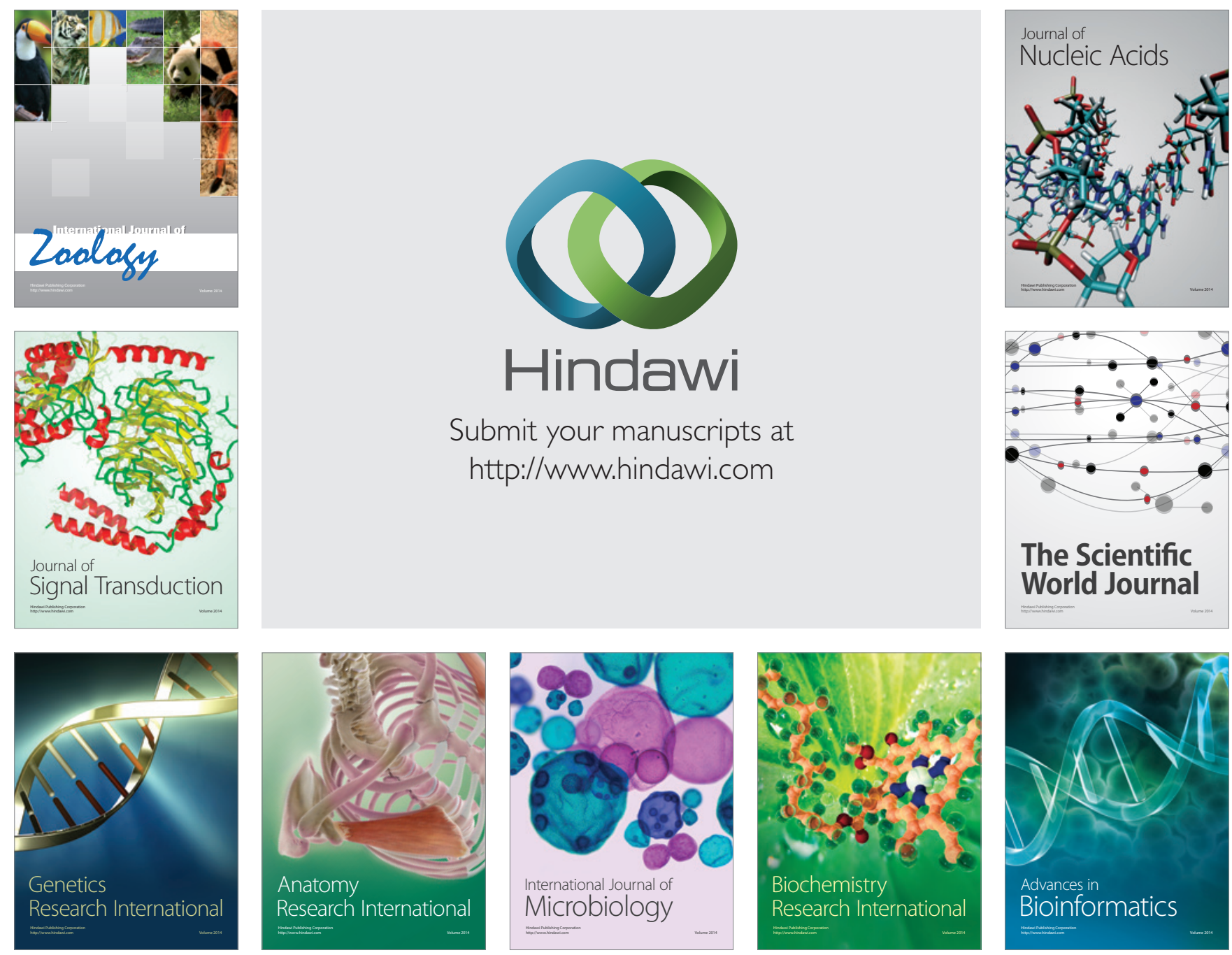

The Scientific World Journal
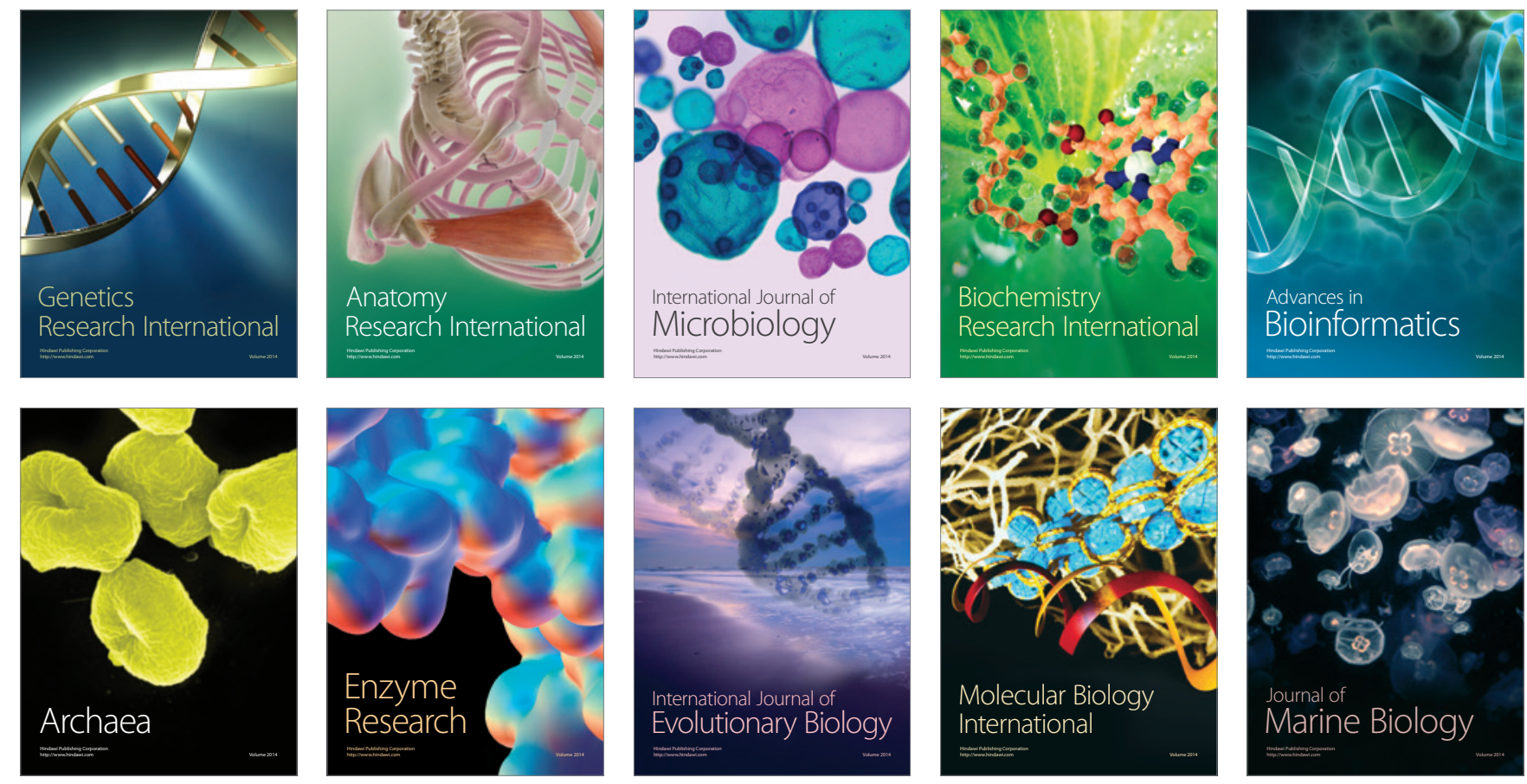